\title{
Estudio Piloto: Influencia de los hábitos orales en el desarrollo de maloclusiones en infantes
}

Pilot Study: Influence of oral habits in the development of malocclusions in infants

\section{Resumen}

El objetivo general del presente estudio fue determinar la influencia de los malos hábitos orales más prevalentes en la oclusión dentaria de infantes que asistieron a la Clínica del Niño de la Facultad de Odontología de la Universidad Nacional Mayor de San Marcos (UNMSM). Por lo que, se realizó este estudio descriptivo y transversal en 50 pacientes infantes que asistieron durante el año 2010 y ya registraban el primer plano de la oclusión. La recolección de los datos se obtuvo mediante encuesta a los padres y examen clínico para el registro de maloclusiones en los infantes. Se aplicó la prueba de Chi cuadrado y $r$ de Pearson (SPSS 17) con un nivel de significancia del $95 \%$ ( $p<0,05)$. En nuestro estudio se observó que existe relación entre la succión digital frecuente con profundidad del paladar en el 60 $\%$ (11 infantes) (p 0.01). Asimismo se observó relación entre el plano terminal y succión digital (p $0.00)$. Se comprobó que existe una relación positiva entre el paladar profundo y el hábito de respiración (p:0.00), siendo la edad de los infantes de 19-36 meses, los que registraron la mayor prevalencia. Los resultados determinaron que la aparición temprana de malos hábitos más prevalentes (succión digital 21 $\%$ y respiración bucal $12 \%$ ) es más evidente entre las edades (19-36 meses) sobre la maloclusión dental. El hábito de succión digital tiene repercusión en la profundidad del paladar así como en la relación del plano terminal recto y distal.

Palabras claves: Maloclusión, hábitos, infantes

\section{Abstract}

The aim of the study was to determine the influence of oral habits more prevalent in the dental occlusion of infants who attended the dental clinic of San Marcos University. This is a descriptive and cross-section study in patients attending the Infant Clinic of the Child, in the 2010. The sample consisted in 50 infants who recorded the first plane of occlusion. The data collection was obtained through a survey to parents and subsequently performed clinical examination for registration of malocclusion in children. They applied the chi-square test of Pearson $r$ (SPSS 17) with a significance level of $95 \%(\mathrm{p}<0.05)$. Our study observed a relation between the common digital suction depth of the palate with $60 \%$ (11 infants) ( $\mathrm{p}$ 0.01). Also was no relation between the plane and finger sucking terminal for within the age range of 19-36 months, also there was a terminal plane (straight or distal) in $89 \%$ (8 infants) in the group with digital suction its $100 \%$ ( 9 infants) (p 0.00). It study was found that a positive relation between deep palate and the habit of mouth breathing, found that $100 \%$ of them ( $p 0.00)$, being the age of the infants of 19-36 months, those who had the highest prevalence. The results showed that early onset most prevalent habits (finger sucking and mouth breathing $21 \% 12 \%$ ) is most apparent between ages (19 - 36 months) on dental malocclusion. The thumb sucking habit has an impact on the depth of the palate and was relation of the rectum terminal plane and distal step.

Keys words: malocclusion, habits, infants.

\section{Introducción}

Actualmente la atención precoz que se realiza en la clínica de bebés es una necesidad y felizmente es una acción factible en nuestra realidad, debido a que cumplimos con uno de los lineamientos emitidos desde el año 2002 por la Organización Mundial de la Salud (OMS), donde enmarca el cuidado de la salud oral en la población de alto riesgo, la cual lo constituyen los infantes y los niños. Una de las principales enfermedades orales, poco estudiadas durante la infancia son las maloclusiones. Se sabe además que existen factores que favorecen a la instalación de esta patología, como son los malos hábitos

\section{Artículo Original}

\author{
María Angélica Alvarez- \\ Páucar ${ }^{1}$, Katherine Quiroz \\ Gonzales ${ }^{2}$, Ana Sari Chamilco \\ Gamarra², César Akira \\ VásquezTokuchi ${ }^{2}$, Lía Luza \\ Yaranga ${ }^{2}$, Jessica Huanca \\ Sánchez ${ }^{2}$, Karla Wieslawa \\ Medina Gutti ${ }^{2}$ y Roxana \\ Delgado Llancari ${ }^{2}$.
}

\begin{abstract}
${ }^{1}$ Docente del Dpto Acad de Estomatología Pediátrica de la Facultad de Odontología, Universidad Nacional Mayor de San Marcos

${ }^{2}$ Estudiantes del 5 to ańo de la Facultad de Odontología, Universidad Nacional Mayor de San Marcos
\end{abstract}

\section{Correspondencia:}

Mg. María Angélica Alvarez Paucar Facultad de Odontología. UNMSM. Av. Germán Amézaga s/n. Lima 1. Perú Teléfono: 999115270

Correo Electrónico:

angelicaalvarez1@hotmail.com orales, que pueden ocasionar desequilibrio en la musculatura estomatognática - facial, estos patrones se aprenden, al tratarse de prácticas o costumbres adquiridas por la repetición frecuente de un mismo acto, en un principio consciente y luego inconsciente por la habituación, como son: respiración nasal, masticación, fonación y deglución, considerados fisiológicos o funcionales; aunque existen también algunos no fisiológicos, como la succión (digital), el uso del chupete, la respiración bucal, la interposición lingual, la onicofagia, la queilofagia y otros.

$\mathrm{Al}$ inicio, cuando la función es alterada, el desvío es imperceptible, sólo se evidencia algún tiempo más tarde, por ejemplo el amamantamiento insatisfactorio, el uso continuo del chupón, la succión digital, la insuficiencia masticatoria, la respiración bucal, como factores de deformación, asimismo cuando se ofrece el chupón para calmar al bebé o se permite que este reciba amamantamiento materno hasta los 2 ó 3 años ó uso del biberón hasta más allá de los 3 ó 4 años y que con diferentes razones los padres contribuyen a que los hábitos se instalen conllevando a desvíos de forma y función. ${ }^{2}$ El periodo de amamantamiento y erupción de los primeros dientes primarios son óptimas ocasiones para diagnosticar y aplicar futuras medidas profilácticas y terapéuticas precoces. ${ }^{2,3}$ 
El presente trabajo busca revelar la prevalencia de los hábitos orales y su relación con la presencia de alteraciones en la oclusión dentaria, manifestando el tipo de maloclusión temprana, que en el futuro se buscará realizar el tratamiento interceptivo de esta anomalía en el momento oportuno.

\section{Material y método}

Este es un estudio descriptivo de corte transversal. Cuya muestra estuvo conformada por 50 niños menores de
47 meses de edad, tomados al azar de los pacientes que acudieron a la Clínica Integral del Niño de la Facultad de Odontología de la UNMSM durante los meses de abril a agosto del 2010 . Asimismo se cumplió con los criterios y normas éticas establecidas. Para la recolección de datos, se confeccionó una ficha, la cual estaba conformada en 02 partes: la primera, una entrevista tipo estructurada a las madres con preguntas relacionadas a los hábitos de succión no nutritiva, hábitos de masticación y disturbios funcionales y la segunda parte una ficha clínica dental donde se registró el examen clínico de los posibles desvíos y maloclusiones, según los momentos de oclusión propias de la dentición temporal. Para dicho estudio se realizó la calibración correspondiente a los 02 examinadores principales. Para el análisis estadístico, fue utilizado el SPSS 17, mediante la prueba de Chi cuadrado y $r$ de Pearson con un nivel de significancia del $95 \%(\mathrm{p}<0,05)$.

\section{Resultados}

El presente gráfico, muestra la prevalencia de los hábitos orales desarrollados en infantes que asisten a la Clínica Odontológica - UNMSM. Donde se puede observar que el uso de biberón, succión digital y respiración bucal son los malos hábitos orales que evidenciaron mayor porcentaje.Ver Fig 1.

Por otro lado, cuando se realizó el examen clínico, la relación dental que manifestaron los infantes según los 04 momentos de la oclusión que propugna Walter (2000) 1 evidenció un alto porcentaje de normalidad. Ver Tabla 1 y 2.

Con lo que respecta al uso del biberón frecuente y tipo de arco superior no se encontró alteración en el componente dental, registrándose un Arco Baume Tipo I, en el 100\% (23 infantes). (p 0.00). Ver Fig 2.

Además, se observó que existe una relación de asociación entre la succión digital con profundidad del paladar, pudiéndose observar que dentro del grupo que presentó succión digital (19 infantes), el $60 \%$ (11 infantes) presentó paladar profundo (p 0.01). Asimismo, se halló que la aparición temprana de la succión digital genera paladar profundo registrándose en $6(85 \%)$ de los 7 infantes $(100 \%)$ de menor edad, antes de los 2 años 5 meses; contrastando con aquellos que adquirían el hábito de succión digital posterior a los 2 años 6 meses de vida en el cual del $100 \%$ que presentó succión digital después de los 29 meses, ninguno desarrollo paladar profundo. (p 0.02).

Cuando se analizó si existe asociación entre la relación del plano terminal y los hábitos orales, se observó una relación muy fuerte, entre el tipo de plano terminal y el hábito de succión digital que presentaron los infantes, Ver Fig 3.

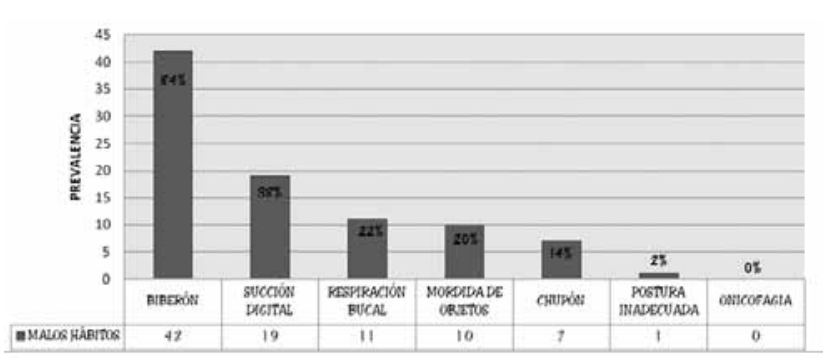

Fig 1. Prevalencia de hábitos orales

Tabla $\mathrm{N}^{\circ} 1$. Relación dental de los infantes

\begin{tabular}{lcccclccccc}
\hline & \multicolumn{2}{c}{ Over-Jet } & \multicolumn{1}{c}{ Rel. Canina } & \multicolumn{3}{c}{ Over - Bite } & \multicolumn{3}{c}{ Rel. Terminal } \\
\hline Relación & $\mathrm{n}$ & $\%$ & $\mathrm{n}$ & $\%$ & Relación & $\mathrm{n}$ & $\%$ & Relación & $\mathrm{n}$ & $\%$ \\
\hline & & & & & & & & & & \\
Clase - Normal & 49 & $48 \%$ & 37 & $74 \%$ & Normal & 29 & $58 \%$ & Esc. Mesial & 21 & $42 \%$ \\
Clasell & 1 & $2 \%$ & 5 & $10 \%$ & Profunda & 21 & $42 \%$ & Esc. Distal & 4 & $8 \%$ \\
Claselll & 0 & $0 \%$ & 3 & $6 \%$ & Abierta & 0 & $0 \%$ & Plano Recto & 10 & $20 \%$ \\
NR & 0 & $0 \%$ & 5 & $10 \%$ & NR & 0 & $0 \%$ & NR & 15 & $30 \%$ \\
\hline Total & 50 & $100 \%$ & 50 & $100 \%$ & Total & 50 & 100 & Total & 50 & $100 \%$ \\
\hline
\end{tabular}

Tabla $N^{\circ}$ 2. Tipo de Arcos

\begin{tabular}{lcccc}
\hline \multicolumn{5}{c}{ Arco de Baume } \\
\hline & \multicolumn{2}{c}{ Maxilar Superior } & \multicolumn{2}{c}{ Maxilar Inferior } \\
\hline Relación & $\mathrm{n}$ & $\%$ & $\mathrm{n}$ & $\%$ \\
\hline Tipo I & 29 & $58 \%$ & 27 & $54 \%$ \\
Tipo II & 19 & $38 \%$ & 20 & $40 \%$ \\
NR & 2 & $4 \%$ & 3 & $6 \%$ \\
Total & 50 & $100 \%$ & 50 & $100 \%$ \\
\hline
\end{tabular}

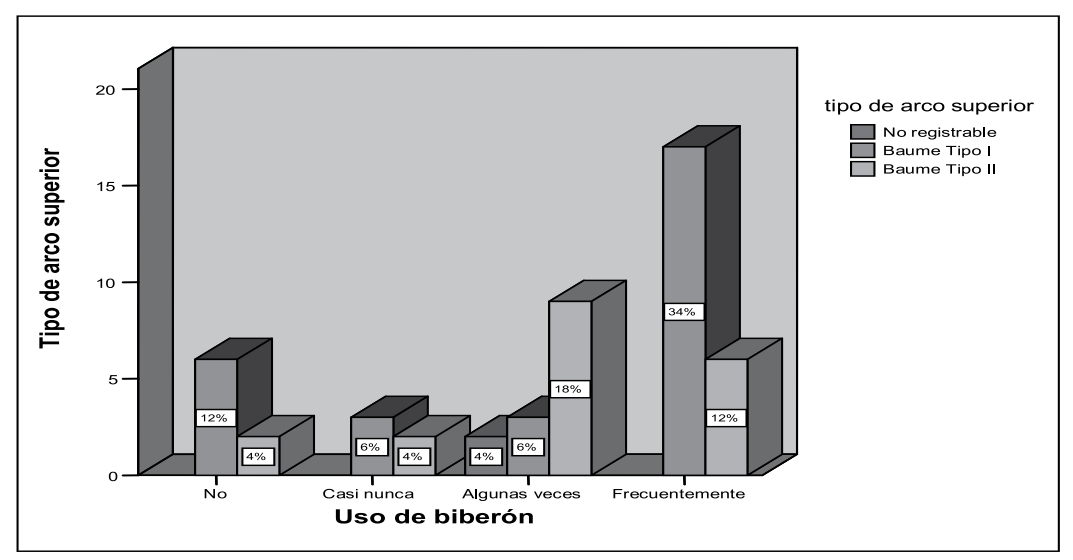

Fig2. Arco de Baume Superior según hábito de uso del biberón 
Ya que dentro del rango de edad de 19-36 meses, se encontró un plano terminal (recto o distal) en un $89 \%$ (8 infantes) dentro del grupo que presentó succión digital en su $100 \%$ (9 infantes). (p 0.00).

Por otro lado, se comprobó que existe una relación positiva entre el paladar profundo y el hábito de respiración bucal, encontrándose que el $100 \%$ de ellos (10 infantes) que presentaba respiración bucal estaba asociado a paladar profundo ( $\mathrm{p}: 0.00$ ), siendo la edad de los infantes que registraron la mayor prevalencia, los infantes de 19- 36 meses.

\section{Discusión}

El presente trabajo evidenció la siguiente secuencia de hábitos orales más prevalentes: uso del biberón como el más frecuente $(84 \%)$, seguido por la succión digital $(38 \%)$ y respiración bucal (22\%); similar al estudio realizado por Agurto y cols (1999) ${ }^{4}$ donde encontraron que el $62 \%$ de la población presentaban el hábito de succión, de los cuales $55 \%$ corresponden a succión de biberón, $23 \%$ a succión digital y el $23 \%$ respiración bucal; sin embrago, la frecuencia de la respiración bucal en el presente trabajo fue superior, respecto al uso del chupón (14\%), similar resultado obtenido por la investigación de Carrasco y cols. $(2005)^{5}$, donde también evidenció un porcentaje bajo. Por otro lado, en el mismo estudio realizado por Carrasco y cols. (2009) ${ }^{5}$ se encontró que el hábito del uso biberón fue de $61.9 \%$, lo cual difiere a la cifras encontradas en el presente trabajo (84\%).

Dentro de las alteraciones de las anomalías verticales el $42 \%$ corresponden a una sobremordida profunda, lo cual difiere con los resultados obtenidos por Alvarez ${ }^{6}$ que mencionan que la anomalía prevalente es la mordida abierta en su estudio y que el presente trabajo no halló. Asimismo, cabe recalcar que el presente trabajo no halló ninguna anomalía transversal entre los infantes examinados.

Del total de la población de estudio un $38 \%$ presentó succión digital, siendo este el hábito de succión no nutritiva más frecuente; por encima del uso de chupete o chupón $(14 \%)$ y de la respiración bucal (22\%). Esta cifra sobre succión digital, se encuentra por encima de otras investigaciones anteriores, que van desde un $15,26 \%$ en el estudio realizado por Fernández-Torres ${ }^{7}$, asimismo el estudio realizado por Agurto

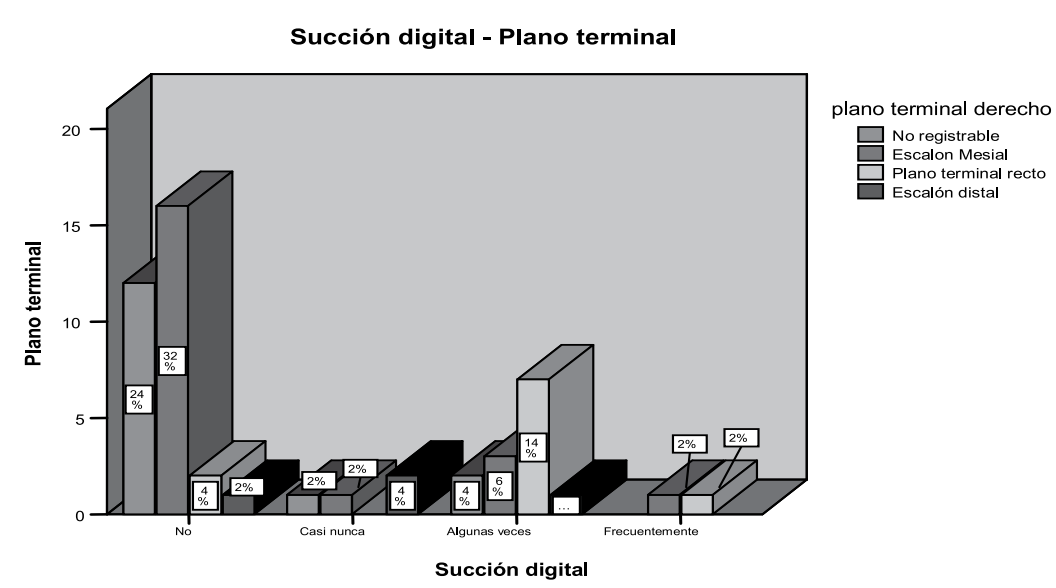

Fig 3. Plano Terminal según el hábito de succión digital

4, donde reflejó un $23 \%$ o el estudio realizado por Vázquez y cols. (2005) 8 que encontró un $21 \%$ de prevalencia de succión digital.

Si bien es cierto que, las mayores deformaciones maxilares y dentarias se observaron en la succión digital, se debe a que el dedo constituye un elemento rígido, no colapsable y está disponible en todo momento según las necesidades de succión del niño ${ }^{2,9}$. Es sabido que las deformaciones dento-esqueletales que producen estos hábitos dependerán de la intensidad y frecuencia de este., ${ }^{2,3,6,10}$ Dentro del grupo que presentó succión digital (38\%), el $60 \%$ presentó paladar profundo como posible consecuencia del mismo; otros estudios similares muestran cifras más bajas como el de Vázquez $(2005)^{8}$ que encontró paladar profundo en un 22,1\% de los niños que presentaban succión digital.

En este estudio se encontró que la mayoría de los niños con frecuente lactancia materna no presentaban hábitos de succión no nutritiva así mismo las investigaciones realizadas por Praetzel y cols. (2002) ${ }^{11}$ y Moimaz (2008) 5 demuestran que la lactancia materna protege contra la instalación de hábitos de succión no nutritiva durante el primer año de vida. Cuando analizamos los medios para la remoción de los hábitos nocivos de succión encontramos varias líneas de pensamiento, la succión es una faceta normal entre los 3 y 4 ańos de edad; el hábito debe ser retirado de inmediato cuando este surja. ${ }^{5}$

Alemán y cols. $(2007)^{10}$ demostraron en su estudio que, de 32 nińos que presentaban succión digital el $53 \%$ desarrollaron plano terminal recto, resultado que coincide con nuestro de estudio, donde hallamos que el 53,3\% de infantes que presentan hábito de succión digital frecuente no presentaban altera- ciones en la relación molar. Por lo tanto se podrían decir que la succión digital no genera un porcentaje significativo de maloclusiones en la relación molar decidua.

\section{Conclusiones}

Existe influencia de los hábitos orales entre ellos: succión digital y respiración bucal con la presencia temprana de los desvíos dentales en el infante como la profundidad del paladar, haciéndose notoria entre las edades de 19 a 36 meses, desarrollado en los infantes sobre la oclusión dentaria en los diferentes momentos de la oclusión. En los infantes que completaron la fórmula primaria se observó una relación terminal: escalón distal y recto en aquellos infante que desarrollaron hábito de succión frecuente. Además se observó que el hábito del uso del biberón frecuente no daba una tendencia marcada para alterar el componente dental con respecto al arco de Baume.

Este trabajo servirá de gran ayuda a los padres y profesionales para la detección temprana de los malos hábitos orales y desarrollar en el futuro un programa preventivo e interceptivo de las maloclusiones en la primera infancia.

\section{Referencias bibliográficas}

11. Ricardo RM. Comportamiento de escolares de primaria con hábito de succión digital luego de una intervención educativa. MEDISAN 2011; 15(2): 222 [Fecha de acceso 19 de Noviembre de 2010]. Disponible en: http://bvs.sld.cu/revistas/ san/vol_15_2_11/san12211.pdf

2. Ferreira CM. Programa preventivo e interceptivo de maloclusiones en la primera infancia. En: Correa NMS. Odontopediatría en la pri- 
mera infancia, Sao Paulo: Livraria Santos Editora, 2009.

3. Walter LR, Ferrelle A, Issao M. Odontología para el bebé:odontopediatría del nacimiento a los tres años. Actualidades Médico Odontológicas Latinoamericanas (AMOLCA), 2000.

4. Agurto VP, Díaz MR, Cádiz DO y Bobenrieth KF. Frecuencia de malos hábitos orales y su asociación con el desarrollo de anomalías dentomaxilares en niños de 3 a 6 años del área Oriente de Santiago. Rev. chil. Pediatr 1999; 70 (6): [Fecha de acceso 19 de Noviembre de 2010]. Disponible en:

ht tp://www.scielo.cl/scielo. php?script $=$ sci_arttext $\&$ pid =S037041061999000600004

5. Carrasco-Loyola M, Villena-Sarmiento R, Pachas- Barrionuevo FM, Sánchez HY. Lactancia materna y hábitos de succión nutritivos y no nutritivos en nińos de 0-71 meses de comunidades urbano marginales del cono norte de
Lima. Rev. estomatol. Hered 2009; 19(2):83-90. [Fecha de acceso 19 de Noviembre de 2010]. Disponible en: http:// www.upch.edu.pe/ faest/old/publica/2009/vol19_n2/ vol19_n2_09_art2.pdf

6. Olguín PA y Quintana SX. Mordida Abierta Anterior en Niños de 2 a 4 Años y Uso de Pacificadores Orales en un Sector de la Comuna de Lo Barnechea. Revista Dental de Chile; 2001; 92(2); 3-8. [En línea]. [Fecha de acceso 5 de abril de 2010]. Disponible en: http://www.revistadentaldechile.cl/temas\%20agosto\%20 2001/PDF\%20AGOST\%202001/ Mordida\%20Abierta\%20Anterior...pdf

7. Fernández CT y Acosta NM. Hábitos deformantes en escolares de primaria. [En línea]. Rev Cubana Ortod 1997;12(2):79-83. [Fecha de acceso 5 de abril de 2010]. Disponible en: http://www.bvs.sld.cu/revistas/ord/vol12_2_97/ord02297.htm

8. Vázquez LM, González LS y Vázquez LA. Alteraciones clínicas buca- les en niños con hábito de succión digital 2005.[En línea] .[Fecha de acceso 5 de abril de 2010]. Disponible en: http://www.bvs.sld.cu/ revistas/mciego/vol11_supl2_05/ articulos/a1_v11_supl205.htm

9. Larson E. Artificial Syucking habits: Etiology, prevalence and effect in occlusion. Am J Orthod 1994; 20: 10-21.

10. Alemán SP, González VD, Díaz OL y Delgado DY. Hábitos bucales deformantes y plano poslácteo en niños de 3 a 5 ańos. Rev $\mathrm{Cu}$ bana Estomatol 2007;44(2). [En línea]. [Fecha de acceso 21 mayo 2010]. Disponible en: http:/www./ scielo.sld.cu/scielo.php?script $=$ sci_ a r t t e x t \& p i d = S 0034 $75072007000200001 \& \operatorname{lng}=$ es \&nr $\mathrm{m}=\mathrm{iso}$

11. Praetzel JR, Saldanha MJQ, Pereira JES e Guimarães MB. Relação entre o tipo de aleitamento e o uso de chupeta. J Bras Odontopediatr Odontol Bebe 2002;5(25):235-40. 